\title{
SN 2010as and Transitional Ib/c Supernovae
}

\author{
Gastón Folatelli \\ Kavli Institute for the Physics and Mathematics of the Universe, \\ Todai Institutes for Advanced Study, the University of Tokyo, \\ Kashiwa, Japan 277-8583 email: gaston.folatelli@ipmu.jp
}

On behalf of the MCSS, the CSP, and collaborators

\begin{abstract}
We present intensive photometric and spectroscopic observations of SN 2010as carried out by the Millennium Center for Supernova Studies (MCSS) and the Carnegie Supernova Project (CSP). The SN belongs to the transitional type Ibc (SN Ibc) that is characterized by the slow appearance of weak helium lines with low expansion velocities. We find a wide variety of photometric properties among otherwise spectroscopically similar SN Ibc. A hydrodynamical model is used to provide physical properties of SN 2010as in comparison with the bolometric light curve and expansion velocity.
\end{abstract}

Keywords. supernovae: general, supernovae: individual (SN 2010as)

\section{Introduction}

SN 2010as was discovered in NGC 6000 on March 19.2 UT by the CHilean Automatic Supernova sEarch (CHASE; Maza et al. 2010) and classified as a type Ibc SN (Stritzinger et al. 2010). Here we compare its observed properties with those of other objects in this transitional class, namely SN 1999ex, SN 2005bf, and SN 2007Y.

\section{Spectroscopic similarities among SN Ibc}

A spectral time series between -10 and +309 days relative to maximum light was obtained for SN 2010as by the MCSS and CSP, with nearly daily coverage before maximum. The pre-maximum spectra are of type Ic, dominated by Ca II and Fe II lines, with possible $\mathrm{H} \alpha$ or $\mathrm{Si}$ II at $\approx 6200 \AA$. Then He I lines develop marking the transition to a SN Ib. Fig. 1 (left) compares the spectra with those of SNe 2005bf (Folatelli et al. 2006) and 2007Y (Stritzinger et al. 2009) at three epochs. The three SNe are very similar before maximum, but then SN 2007Y develops strong He I lines. The other two SNe remain similar, with SN 2010as showing stronger Ca II features. An additional similarity is the peculiarly low He I line velocities observed at all times (see Fig. 1, right).
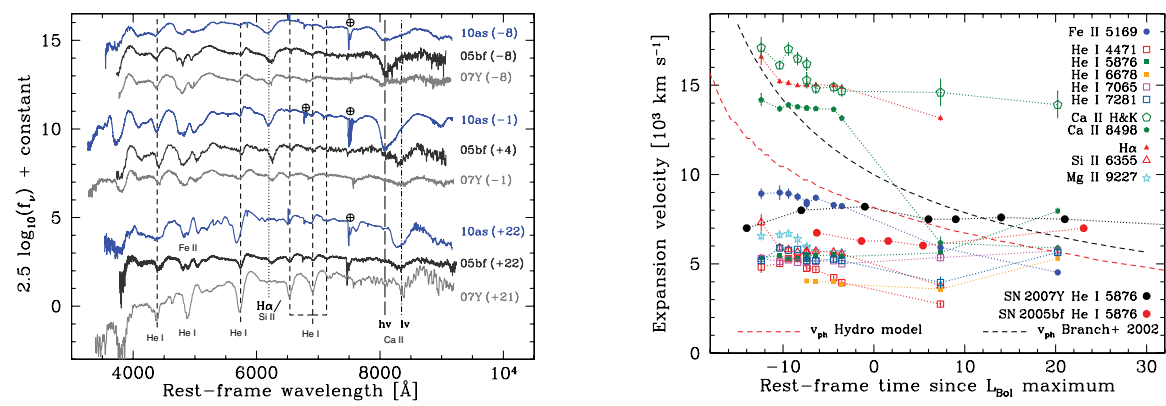

Figure 1. (Left) Spectral comparison with SNe Ibc 2005bf and 2007Y at three different epochs. (Right) Expansion velocities measured from spectral lines (dots) and models (lines). 
Table 1. Absolute peak magnitudes of selected SN Ibc.

\begin{tabular}{lcccc}
\hline SN & $M_{B}$ & $M_{V}$ & $M_{J}$ & $M_{K}$ \\
\hline $2010 \mathrm{as}$ & $-18.1 \pm 0.5$ & $-18.5 \pm 0.4$ & $-18.4 \pm 0.3$ & $-18.2 \pm 0.3$ \\
$1999 \mathrm{ex}$ & $-17.4 \pm 0.3$ & $-17.9 \pm 0.3$ & & \\
$2005 \mathrm{bf}$ & $-18.5 \pm 0.3$ & $-18.6 \pm 0.3$ & & \\
$2007 \mathrm{Y}$ & $-16.2 \pm 0.6$ & $-16.4 \pm 0.6$ & $-16.7 \pm 0.6$ & $-17.0 \pm 1.0$ \\
\hline
\end{tabular}
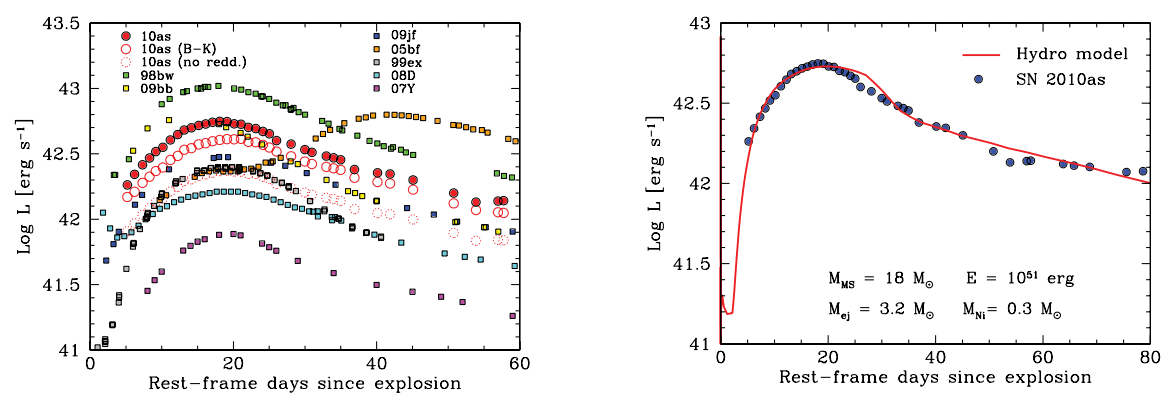

Figure 2. (Left) Bolometric light curves of core-collapse SNe including some SN Ibc. (Right) Bolometric luminosity for a model (solid line) with the indicated physical parameters, compared with the data (dots).

\section{Photometric variety}

Follow-up of SN 2010as was obtained in $B V R I g^{\prime} r^{\prime} i^{\prime} z^{\prime}$ bands at CTIO, and in $J H K$ bands at ESO. The SN was caught 10 days before $B$-band maximum light and followed for over 100 days. Observed colors corrected for Galactic reddening were compared with those of a sample of SNe Ib and Ic from the CSP (Stritzinger et al., in prep.) to derive a host-galaxy reddening of $E(B-V)_{\text {host }}=0.35$ mag $\dagger$. Adopting a distance of $32.7 \pm 3.6 \mathrm{Mpc}$ to the host (NED), reddening-free absolute peak magnitudes of SN 2010as are relatively luminous as compared with other SN Ibc (see Table 1).

A bolometric light curve was obtained by integrating the extinction-corrected optical flux and adding the extrapolation of a black-body fit toward the IR, and a straight line in the UV from the $B$-band point to zero flux at $2000 \AA$. The resulting peak luminosity of $L_{\mathrm{Bol}}=6.3 \times 10^{42} \mathrm{erg} \mathrm{s}^{-1}$ is comparable with that of the peculiar SN 2005bf, although the total radiated energy is much larger for the latter SN. A hydrodynamical model (Bersten et al. 2011) was computed to reproduce the bolometric luminosity (see assumed physical parameters in the right panel of Fig. 2). A relatively large amount of ${ }^{56} \mathrm{Ni}, M_{\mathrm{Ni}}=0.3$ $M_{\odot}$, is required to explain the luminous peak. The explosion energy of $E=10^{51} \mathrm{erg}$ slightly overestimates the measured expansion velocities (see right panel of Fig. 1).

\section{References}

Bersten, M. C., et al. 2011, ApJ, 729, 61

Folatelli, G., et al. 2006, ApJ, 641, 1039

Maza, J., et al. 2010, CBET, 2215, 1

Stritzinger, M., et al. 2009, ApJ, 696, 713

Stritzinger, M., et al. 2010, CBET, 2221, 1

Stritzinger, M., et al. 2013 in prep.

$\dagger$ In agreement with the measured equivalent width of Na I D of $2.1 \AA$. 